\title{
Spatial distribution of DDT and its metabolites in soils from Indoor Residual Spraying in Tshilamusi village, Limpopo
}

\author{
Wilson Gitari ${ }^{1}$, Tonderai Makoni ${ }^{1}$, Vernon Somerset ${ }^{2}$ and Omotola Babajide ${ }^{3}$ \\ ${ }^{1}$ Environmental Remediation and Water Pollution Chemistry Research Group, Department of Ecology and \\ Resources Management, University of Venda, Private bag X5050, Thohoyandou, 0950, South Africa, Tel: \\ +27159628572 , \\ ${ }^{2}$ Department of Chemistry, Cape Peninsula University of Technology, Bellville 7535, Capetown, South Africa \\ ${ }^{3}$ Department of Mechanical Engineering, Cape Peninsula University of Technology, Bellville 7535. Capetown, \\ South Africa: \\ Correspondence: babajideo@cput.ac.za; Tel.: +27219596063
}

\begin{abstract}
The continued application of organochlorine pesticides (OCPs) in indoor residual spraying has posed significant threat to human health in Northern part of South Africa, despite its ban. In this study, we investigated the occurrence and spatial distribution of DDTs and its metabolites in surface soil (30 samples) collected in and around the spray homesteads; demarcated into three concentric zones A, B, C in Tshilamusi Vhembe district, South Africa. DDTs were the most abundant of all the OCPs chemicals found in soil samples. The concentrations of DDT range from 12.19 to $65.69 \mu \mathrm{g} / \mathrm{kg}$, with the highest occurring at zone A which is the zone of application. DDTs and all its metabolites considered in this study were found in appreciable concentrations in soil of Zones B and C, far from spray sites. The results showed a strong correlation between distance and concentration levels of DDTs and its metabolites. However, there is a need for proper monitoring of OCPs chemicals in other environmental matrices as well as relevant agencies enforcing strict adherence to regulations on consumption of OCPs.
\end{abstract}

Keywords: Application; organochlorine; pesticides; metabolites; homesteads; soils

\subsection{Introduction}

The contamination of the environment with organochlorine pesticides (OCPs) have continued to pose significant threat to human health due to their toxicity, stability and resistant to degradation $[1,2,3]$. Despite the ban imposedat the Stockholm Convention on consumption, production, import and export of OCPs since 1970, its use has been prevalent in many countries [4-7]. Dichlorodiphenyltrichloroethane (DDT) has increasingly polluted the environment been widely used in most developing countries mainly for the control of vector diseases like malaria [8].Vhembe district Limpopo is one of such places in South Africa, where there is a high prevalence of malaria vectors like Anopheles funestus and Anopheles arubiensis [9]. Indoor Residual Spray (IRS) or DDT containing insecticides has been widely applied on walls and roofs of traditional unpainted mud-walled thatched huts [10].Studies by Tao et al.[11] have shown that DDT is mainly exposed to humans through food ingestion. The first step in the food chain accumulation would be the uptake of DDT by the roots of plants [12]. Taking into consideration that the majority of the people residing in Vhembe District rely on vegetables cultivated close to their homesteads, which are susceptible to contamination by DDT and its metabolites. There has been little research on the spatial distribution of DDT and its metabolites from sprayed areas as such study would allow for 
better understanding of how DDT is transported to cause harm to human beings and other terrestrial animals. DDT and its metabolites also have different toxic effects and their distribution in the ecosystem is also crucial in knowing how different organisms get to ingest the toxic metabolites.This study therefore aims to investigate the occurrence and spatial distribution of DDT and its metabolites in and around the sprayed homesteads in Vhembe district, Limpopo, South Africa. The findings will expand our knowledge on POPs contamination and provide useful information to local policy makers on indigenous environmental management.

\subsection{Materials and Methods}

\subsection{Study Area}

Tshilamusi area in Mutale, Vhembe district, Limpopo, South Africa (Figure 1) was selected for this study because it is an isolated area. Soil, water and plant samples were collected within a radius of $30 \mathrm{~m}$ in few homesteads divided into three concentric circles; with $A$ being the area of heavy application, $B$ is the area just outside the first zone and $C$ is the outer core were no application of DDT takes place.

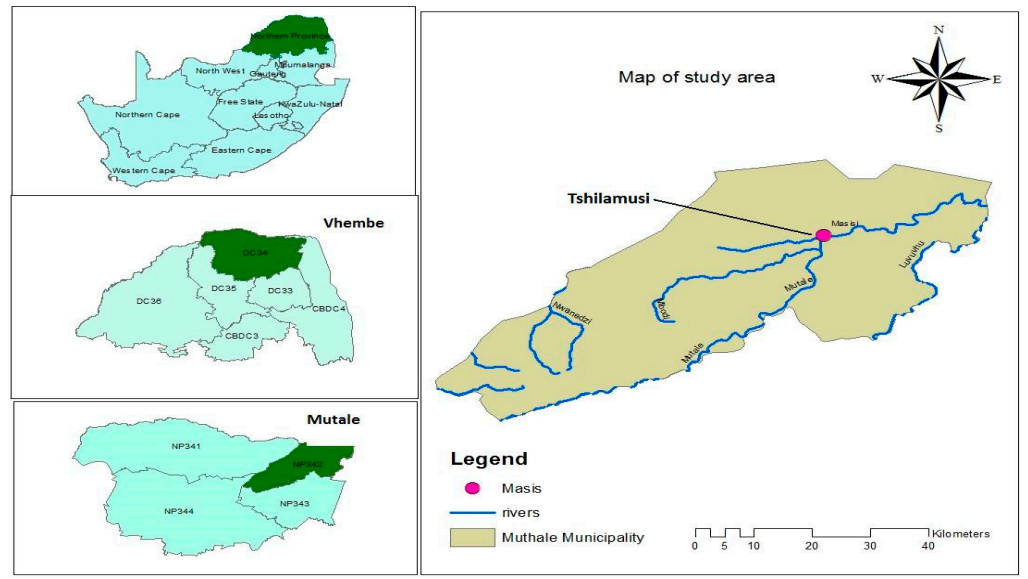

Figure 1: Map of Tshilamusi area in Limpopo, South Africa

\subsection{Sample Collection}

30 surface soil samples were collected evenly around the concentric zone, 5 samples were collected at a $2 \mathrm{~m} \times 2 \mathrm{~m}^{2}$ plot (located on the crossing diagonals four in the corners and one in the crossing point and thoroughly mixed to form a composite sample [13]. The soil samples were collected using stainless steel scoops at depths $0-15 \mathrm{~cm}$ and they were stored in pre-cleaned amber glass containers. Thereafter, they were sieved through a $2 \mathrm{~mm}$ mesh, wrapped in aluminium foil to prevent any reactions and contamination and stored at $4^{\circ} \mathrm{C}$ until further analysis.

\subsection{Sample Extraction and Clean-up}

All solvent were purchased as analytical grades from Fischer scientific, USA. Organochlorine pesticides (OCP) standards, 2,4,5,6-tetrachloro-m-xylene (TCmX), decachlorobiphenyl (PCB 209) and 2, 2', 6, 6'-tetrachlorobiphenyl (PCB 54) were purchased from Ultra scientific, USA. 
All glassware was rinsed in $\mathrm{K}_{2} \mathrm{Cr}_{2} \mathrm{O}_{7}-\mathrm{H}_{2} \mathrm{SO}_{4}$ solution for $24 \mathrm{~h}$ for proper cleansing and drying prior to experimentation. About $10 \mathrm{~g}$ of well dried and homogenized soil samples were extracted with Soxhlet apparatus. The OCP reagents were extracted with dichloromethane (DCM) for $24 \mathrm{~h}$. Surrogate standard of $20 \mathrm{ng}$ each of TCmX and PCB 209 were added to the reagent before extraction. Small granules of copper were added to the extraction flasks to remove elemental sulphur. The extract was concentrated to about 2-3 $\mathrm{mL}$ by a rotator evaporator, after which it was cleansed by alumina/silica column. The column was packed with neutral alumina ( $3 \mathrm{~cm}, 3 \%$ deactivated), neutral silica gel $(3 \mathrm{~cm})$ and anhydrous sodium sulphate $(1 \mathrm{~cm})$. This was followed by elution of the column with $30 \mathrm{~mL} \mathrm{DCM} /$ hexane $(1: 1)$. The eluted fraction was concentrated and reduced to $0.2 \mathrm{~mL}$ using gentle nitrogen stream. A known quantity (20 ng) of PCB-54 was added as an internal standard prior to GC-ECD analysis.

\subsection{Results and Discussion}

\subsection{Concentrations of DDT and its metabolites in soil of Tshilamusi}

The average concentrations of DDT and its metabolites analyzed in the soil samples collected from Tshilamusi area are plotted in Figure 2. The highest concentrations of DDT and its metabolites were found in zone A. Overall, the average concentrations of 4, 4'-DDT was greater than the concentrations of other metabolites in each of the three zones. The high percentage of total DDT occurring as 4, 4'-DDT in soils might be an indication that the DDT in these soils was just recently deposited [11].Yadavet al.[14] also reported higher DDT concentrations in agricultural soils of Nepal; this is because large quantities of DDT are used in agricultural activities than other metabolitesand that it has lower tendencies to biodegrade [15]. Two metabolites 4,4'-DDT and 4,4'-DDE show a decline in concentrations from zone $A$ to $C$, the same cannot be said of 4,4'-DDD which had its highest mean concentration levels in zone $B$ followed by zone $A$ then very low concentrations in zone $C$. In zones $A$ and $B$ the mean concentration of $4,4^{\prime}$-DDT was approximately twice that of $4,4^{\prime}$ DDD and $4,4^{\prime}$-DDE but in zone $C$ there was little difference in concentration between the three metabolites.

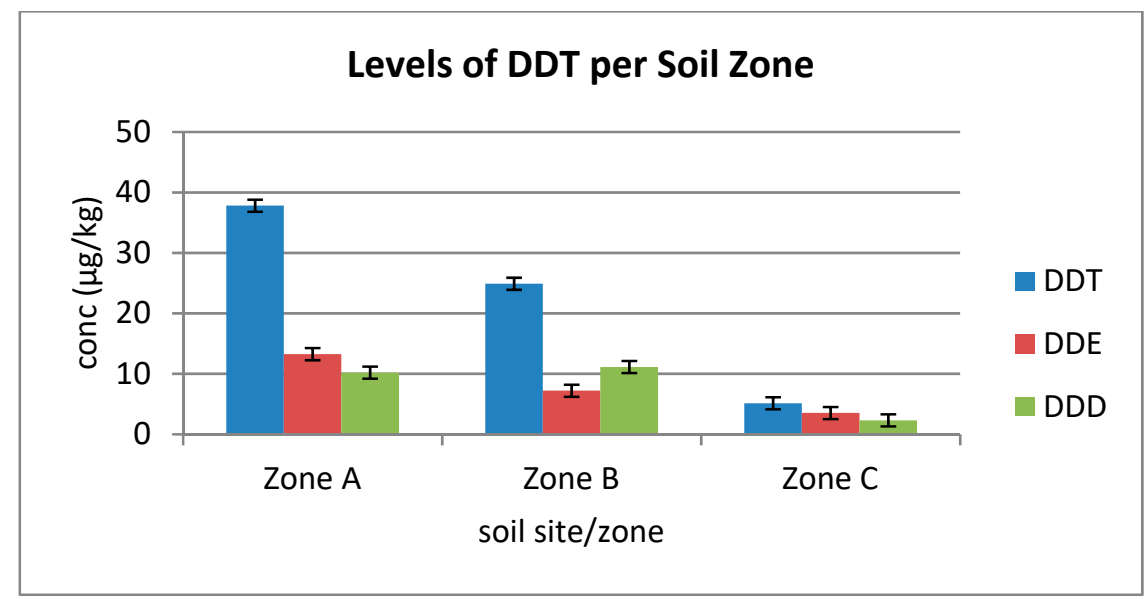

Figure 2. Concentrations of different metabolites of DDT per Soil Zone

Though the levels of $4,4^{\prime}$-DDE were lower than the other metabolites in some of the samples, it is important to note that this metabolite is the most harmful of them all. It has the unique property of existing in the environment for a very long time and has a tendency 
to bioaccumulation in the food web. This means that as we go higher up the food chain as more people eat the chicken which feed on plants and soil the concentration of 4,4'-DDE tends to increase in humans and animal. Studies carried out by $[12,16,17]$ on humans showed higher concentrations of these metabolites than the other metabolites of DDT higher up the food chain.The statistical breakdown and analysis of the LDDT of samples from zones A, B and C are depicted in Table 1. The DDT metabolites were detected in all of the samples in Zone $A$ and zone $B$ and there was $90 \%$ detection in zone $C$. When comparing the three zones zone $A$ had the highest maximum concentrations of DDT detected, which $185.82 \mu \mathrm{g} / \mathrm{Kg}$ was followed by zone B; with $127.97 \mu \mathrm{g} / \mathrm{Kg}$ and zone $\mathrm{C}$ with $27.28 \mu \mathrm{g} / \mathrm{Kg}$, in that order. The mean concentration of DDT $(65.69 \mu \mathrm{g} / \mathrm{Kg})$ was observed to be the highest In zone $A$, which was the area of heavy application of DDT; followed by zone $B$ and $C$ which had $43.23 \mu \mathrm{g} / \mathrm{Kg}$ and $12.18 \mu \mathrm{g} / \mathrm{Kg}$ respectively. This can be attributed to the fact that since $A$ is the zone of application some of the DDT as dust or particles fall from the treated walls or roofs of the dwelling onto the ground.

Table 1 Statistical breakdown of the occurrence and concentrations of 4,4'-DDT, 4,4'-DDD and $4,4^{\prime}-\mathrm{DDE}(\mu \mathrm{g} / \mathrm{Kg})$ in soils of Tshilamusi homesteads

\begin{tabular}{|l|l|l|l|}
\hline & Zone A & Zone B & Zone C \\
\hline $\mathbf{N}$ & 10 & 10 & 9 \\
\hline Min & 4.083 & 3.99 & 3.074 \\
\hline Max & 185.82 & 127.97 & 27.277 \\
\hline Mean & 65.6906 & 43.2331 & 12.18878 \\
\hline Std. Error & 18.17085 & 14.27981 & 2.476656 \\
\hline Stand. Dev & 57.46128 & 45.15672 & 7.429968 \\
\hline Median & 73.5075 & 29.0485 & 13.896 \\
\hline
\end{tabular}

The standard deviation follows the same trend as that of the mean concentrations with zone A having the highest SD of $57.46 \mu \mathrm{g} / \mathrm{Kg}$ followed by zone B with $45.16 \mu \mathrm{g} / \mathrm{Kg}$ and zone $C$ had the lowest SD of $7.43 \mu \mathrm{g} / \mathrm{Kg}$. The 4,4-DDT concentrations detected from soils in Tshilamusi homesteads exhibited a larger standard deviation when compared to the other metabolites due to the higher values which were detected. The higher SD values could be as a result of dissimilar 4, 4'-DDT breakdown and transformation after spraying [10]. The noted variability of 4,4'-DDE may compound to the accumulated dispersion emanating from the dissimilar distribution of DDT due to IRS and from compound transformation.The results of a one-way Analysis of Variance (ANOVA) conducted at 0.05 level of significance revealed very significant variations among the different DDT metabolites observed in each zones. For Zone $A$, the $P$ value is 0.0066 , considered very significant. Variation among different DDT metabolite means/averages is significantly greater than expected by chance. In Zone B the $P$ value is 0.062 , considered not quite significant. Variation among different DDT variant means/averages is not significantly greater than expected by chance. For Zone $C$, the $P$ value is 0.16 , considered not significant which means variation among different DDT metabolite means/averages is not significantly greater than expected by chance. The persistence in the environment of these organochlorines have also been reported in other areas, for instance in China, 4,4'-DDE and 4,4'-DDD have been reported to be widely distributed in soil despite the discontinued use of the pesticide in 1983 [12]. In this study of Tshilamusi homesteads, concentrations of the most dominant metabolite 4,4'-DDT ranged from 0 to $125 \mu \mathrm{g} / \mathrm{Kg}$. 


\subsection{Distribution of DDT and its Metabolites in Tshilamusi Homesteads}

The spatial distribution of DDT sprayed homesteads occured majorly in zone A as shown at the centre of Figure 3. This illustrates that the distribution of DDT was mainly high at the centre around sample $A 5, A 6$ as this was the area of heavy application which was dipicted by the red colour. Moving outwards in zone $B$ there was a general decrease in concentrations except around B3 to the north west were concentrations between 87.00$127.00 \mu \mathrm{g} / \mathrm{kg}$ were also detected. To the east of zone $B$ the concentration decreased rapidly as shown by the blue colour to concentrations below $18.55 \mu \mathrm{g} / \mathrm{Kg}$. Moving on to the south east the DDT concentrations in zone B were slightly higher than the one on the eastern side but at the same time lower than in zone A. In Zone $C$, the majority of the area had concentrations lower than $8 \mu \mathrm{g} / \mathrm{Kg}$ and in some cases as low as between $0-1.98 \mu \mathrm{g} / \mathrm{Kg}$. The northwest zone $\mathrm{C}$ gave high concentrations detected by the brownish colour which represents concentrations between $27-40.9 \mu \mathrm{g} / \mathrm{Kg}$. There was a general decrease in concentration with distance from zone $A$ which was the zone of application. A comparative analysis of the concentrations of DDT in the three zones revealed that zone $A$ had higher concentrations of $4,4^{\prime}$-DDT. The proportions of total DDT occurring as $4,4^{\prime}$-DDD and $4,4^{\prime}$ DDE could indicate site to site differences in the patterns of DDT breakdown [18].For the variation of total DDT levels in the three zones a One-way Analysis of Variance (ANOVA) was used to check the variation of the total DDT in the three soil Zones (A, B and C) at 0.05 level of significance. The $P$ value was 0.0258 , considered a significant variation among total DDT Zones. It is important to note that all the 3 metabolites were detected in the three zones, so this means that there is high probability of the DDT getting into the food web or affecting humans and animals [19]. These results from Tshilamusi raise concerns regarding human exposure to the soils which are contaminated and most notably to children. Butte[20] reported that the exposure could be as a result of ingestion of non-food items and regular hand to mouth contact. In a rural area like Tshilamusi with no pavements, small children spend most of their time outdoors playing and even eating the soil during games hence there is high risk of exposure.Once DDT has been sprayed on the walls of the huts it is now subject to different agents of weather and different physiochemical reactions which will determine the distance and rate of travel from the area of application [14]. In this study, there was a huge difference in the total DDT detected in each of the 3 zones, with Zone $A$ as expected having the highest concentrations followed by $B$ then $C$. This can be attributed to the obvious fact that more DDT is found in the zone wherein its sprayed yearly and also the other reason is the fact that once it is in the soil it will required processed such as erosion, dispersion and heavy winds to move the DDT into other zones [21]. These processes lead to the conclusion that pesticide movement in the environment is very complex because transfers in to different environmental compartments are always occurring continually all the time. Although long range transportation of pesticides is a possibility it does not necessarily mean that all pesticides sprayed will travel long distances or that they will end up in ground water [22]. This is true when we look at the Thsilamusi homesteads wherein the majority of the DDT was detected in the first two zones with the outer zone contributing about $8 \%$ to the total DDT detected in all the zones. 


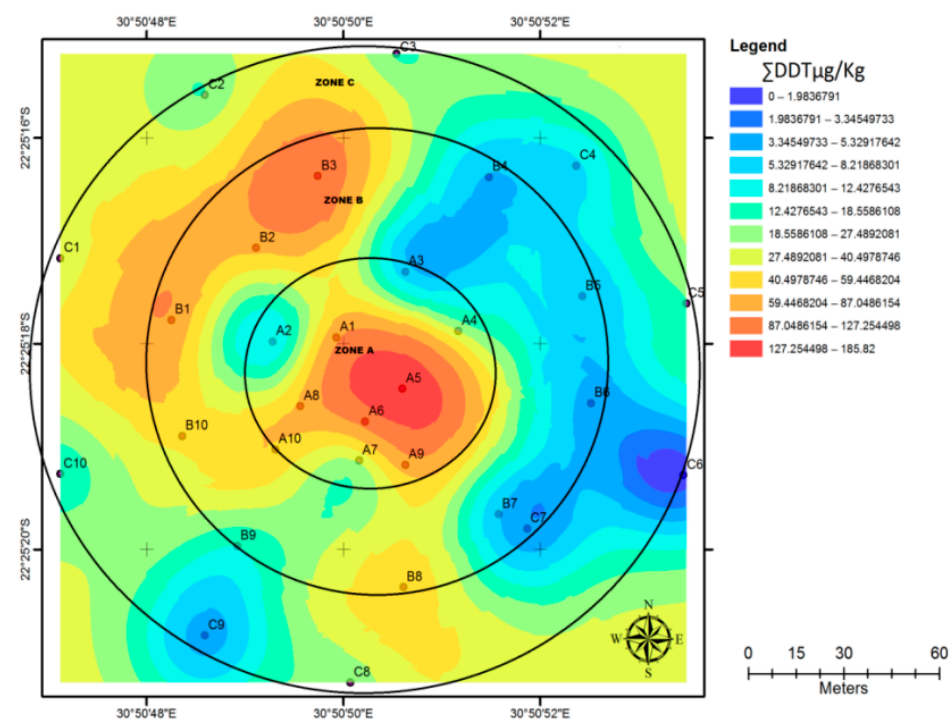

Figure 3: Map showing the zones and distribution of LDDT in the soils of Tshilamusi.will this diagram be regible, you need to magnify it abit to be regible

In Tshilamusi homesteads notable concentrations of 4,4'-DDT, 4,4'-DDE and 4,4'-DDD were detected far away from the area of application in zone $C$. [13] discovered that even if the pesticides are not volatile, the longer time that they persist might result, over time in measureable quantities of the pesticide moving through the atmosphere and accumulating in the unsprayed areas.Several studies have pointed out the fact that pesticides regardless of the medium that they are applied in all have the potential to be transported by air [23]. In Tshilamusi area, the local folks usually sweep their huts and the surrounding area and this dust can be carried over distances by wind hence landing in places where DDT was not originally sprayed.

\subsection{Conclusion}

The concentrations of the 4, 4'-DDT DDT metabolites being the most dominant in soil samples might lead to further exposure to children and adults who come into contact with the soil. Although the concentrations of pesticide residue reported under this investigation were low, it is crucial to note that soil pollution can play a vital role in the global fate and distribution of pesticides. Soil can act as a sink for pesticides from which they can be released into water or air and maybe taken up by plant roots and bioaccumulate in the food chain to an extent higher enough to cause chronic adverse effects to consumers. The detection of DDT and its metabolites in Zone C, far away from the area of spraying is of concern because these metabolites have a longer half life leading to more accumulation of the compound. Detection in soil means that there is a possibility of DDT bioaccumulating in the food chain.It is important to note that the quotient DDT/DDE in all matrices was always $>1$ thus the need for more research and analysis with respect to its effect on the Tshilamusi people. The need to initiate risk-reduction programmes which reduce exposure to the pesticide in this area is urgently required. The study findings would help in enforcing safety regulations for Indoor Residual Spraying. 
Acknowledgments:The authors would like acknowledge the University of Venda, Directorate of Research and Innovation for funding the MSc project that resulted in this publication

\section{References}

1. Jiang, Y. F., Wang, F., Jia, Y., Wu, M. H.., Shang, G. Y., Fu, J. M. Levels, composition profiles and sources of polycylic hydrocarbons in urban soil of Shangai, China. Chemosphere2009 75, 112-118.

2. Li, Y., Haibo, Z., Qingbo, L., Qian, Z., Xianobing, C., Chen, L. Characteristics of residual organochlorine pesticides in soils under different land-use types on a coastal plain of the yellow River Delta. Environ. Geochem. Health 2016 38,535-547.

3. Jones, K. C., de Voogt, P. Pesrsistent organic pollutants (POPs): state of science. Environ. Pollut. 1999100 (1-3), 2009-221.

4. Ge, I. Woodward, L. A., Li, Q. X., Wang, J. I. Composition, distribution and risk assessment of organochlorine pesticides in soils from the Midway Atoll, North pacific Ocean. Sci. Total Environ. 2013 452, 421-426.

5. Yang, Y., Yun, X., Liu, M., Jiang, Y., Li, Q. X., Wang, I. Concentrations, distributions source and risk assessment of organochlorine pesticides in surface water of the East lake, China. Environ. Sci. Pollut. Res. 2014 21, 3041-3050.

6. Whitehead, T.P., Cruspo Smith, S. M., Park, J-S., Petreas, M. X., Rappaport, S. M., Metayer, C. Concentrations of persistent organic pollutants in California children's whole blood and residual dust. Environ. Sci. Technol. 201549 (15), 9331-9340

7. Yadav, I. C., Devi, N. L., Li, I., Zhang, G., Shakya, P. R.Occurence, profile and spatial distribution of organochlorines pesticides in soil of Nepal: Implication for source apportionment. Sci. Total Environ 201609133

8. Dalvie MA, Myers JE, LouTM, Robins T, Omar S, Riebow J. Exploration of different methods for measuring DDT exposure amongst malaria vector control workers in Limpopo Province, South Africa. Environ Res; 2004 96: 20-27.

9. Barnhoorn, I.E.J. Bornman MS, Van Rensburg C and Bouwman H. DDT residues in water, sediment, domestic and indigenous biota from a currently DDT-sprayed area. Chemosphere, 2009 08: 045doi:10.1016/j.chemosphere

10. WHO 2002. Environmental Health Criteria: Endoslufan, World Health Organization, Geneva

11. Tao S, Liu XW, Li QX, Zhou DX, Li X, Yang YF, Yue DP. Organochlorine pesticides residues in chicken and eggs at a poultry farm in Beijing China. Env Poll 2009 157(2): 497-502.

12. Van Dyk (2010) DDT contamination from indoor residual spraying from Malaria Control, Science of Total Environment 2010408: 2745-2752.

13. Gong ZM, Tao S, Xu FL, Liu XW. Level and distribution of DDT in surface soils from Tianjin, China. Chemosphere 2004 54: 1247-1253.

14. Zhang, Q., Zhaojing, C., Yingming, L., Pu, W., Chaofei, Z., Guanjun, G., Ke, X., huizhong, S., Schuchang, Z., yang, L., Guibin, I. Occurrence of organochlorine pesticides in the environment matrices from king George Island, west Antarctica. Environ.Pollut. 2015 206, 142-149.

15. Loganathan, B. G., Kannan, K. Global organochlorine contamination trends: an overview. Ambio 1994 23, 187-191. 
16. Barnhoorn, I.E.J. Bornman MS, Van Rensburg $\mathrm{C}$ and Bouwman $\mathrm{H}$. DDT residues in water, sediment, domestic and indigenous biota from a currently DDT-sprayed area. Chemosphere, 2009 8:45 doi:10.1016/j.chemosphere.2009.08.045

17. Dalvie MA, Myers JE, Lou TM, Robins T, Omar S, Riebow J. Exploration of different methods for measuring DDT exposure amongst malaria vector control workers in Limpopo Province, South Africa. Environ Res; 2004 96: 20-27.

18. Phillips RS. Current status of malaria and potential for control. ClinMicrobiol Rev 2001 14:208-226.

19. Cheung, K.C., Leung, H.M., Kong, K.Y.,Wong, M.H. Residual levels of DDTs andPAHs in freshwater and marine fish from Hong Kong markets and their health risk assessment. Chemosphere 2007 66, 460-468.

20. Butte W (2004). Sources and impacts of pesticides in indoor air environment. The handbook ofenvironmental chemistry, 4. Berlin: Springer-Verlag F; p. 89-116.

21. Pozo K, HarnerT, Lee CS, Sinha KR. Assessing seasonal and spatial trends of POP in Indian agricultural regions using PUF disk passive air samples: Environmental Pollution 2011 159: 646-653.

22. Kwong R.M.W, Yu KN, Lam KS, Wang W. Uptake, elimination and biotransformation of aqueous and dietary DDT in marine fish. Env Toxic Chemistry 2008 27. No 10: 2053-2063.

23. Dalla Villa R, de CarvalhoDores ERG, CarboL, Cunha MLF. Dissipation of DDT in a heavily contaminated soil in Mato Grosso, Brazil. Chemosphere 2006 64:549-554. 